\title{
Las redes sociales de Internet: también dentro de los hábitos de los estudiantes universitarios
}

\author{
al \\ Eucario Parra Castrillón ${ }^{* *}$
}

Recibido: 16 de agosto de 2010 Aceptado: 18 de septiembre de 2010

\begin{abstract}
Resumen
Analizar la pertenencia de los jóvenes universitarios a las redes sociales de Internet es un asunto que advierte tres conceptos fundamentalmente: la existencia de la sociedad red incorporada a la realidad junto con otras presencias como las expresadas en la naturaleza y en la vida de ciudad; la cercanía del ciberespacio, en el mismo contorno de la plaza pública, la institución educativa, la familia o el trabajo; y las potencialidades de Internet como generador de satisfactores para algunas necesidades humanas.
\end{abstract}

Estas realidades justifican la atracción de las redes sociales. Aspectos como la presencia transcultural o la omnipresencia hacen que las redes se comprometan como nodos productores y consumidores de información. A esto se suma que en estas redes las personas fácilmente pueden expresarse, ser escuchadas, compartir identidades, deleitarse con afinidades comunes. Por supuesto, no puede darse por sentado que sean ambientes perniciosos o que por sí mismas tales comunidades sean triviales. De hecho, parte de la convivencia en la red virtual es una extensión de las relaciones sociales cotidianas.

Tampoco puede deducirse que por su acceso libre y fácil, las redes sociales se relacionen directamente con los peligros para la integridad de las personas y que estar allí sea ya el camino para abusar y ser abusado. Anterior a la estadía en redes, está la educación recibida de la familia, la escuela, el barrio. Ir en Internet hacia un comino u otro son opciones surgidas en los propios valores de las personas.

La eclosión de las redes sociales como punto de encuentro entre las personas determina temores y juicios, a veces apresurados. Es así como llega a advertirse que para los estudiantes universitarios, no son una buena opción, cuando en realidad para este público la red es también atractiva. Estos no van a las redes para sus indagaciones académicas, pero, sin que se vengan a menos éstas, sí van para fines personales y en búsqueda de satisfactores a algunas de sus necesidades.

Palabras clave: infovirtual, mundo virtual, red social, sociedad red, virtualidad.

Este artículo de investigación científica y tecnológica hace parte de la investigación Las redes sociales de Internet: también dentro de los hábitos de los estudiantes universitarios.

Título profesional en Ingeniería de Sistemas y postgrados de, maestría en Educación y maestría en Software Libre. Medellín, Colombia. Correo electrónico: investigacionesinfo@tdea.edu.co 


\title{
Internet social networks, also a habit of university students
}

\begin{abstract}
Analyze the university students belonging to social networks on the Internet is an issue that says three fundamental concepts: the existence of built-in network society to reality along with other presences as expressed in nature and city life; the proximity of cyberspace in the same shape of the public square, school, family or work, and the potential of Internet as a generator of satisfaction for some human needs.
\end{abstract}

These realities justify the attraction of social networking. Aspects such as the cross or the ubiquitous presence cause the nodes to commit as producers and consumers of information. Added to this is that these networks people can easily express themselves, be heard, share identities with common delight. Of course, it can be assumed to be harmful environments or that such communities themselves are trivial. In fact part of the coexistence in the virtual network is an extension of everyday social relations.

Nor can it be inferred that its free and easy access, social networks are directly related to the dangers to the integrity of people and that being there is and the way to abuse and be abused. Previous to stay in networks that education received from family, school, neighborhood. Go online to a straw or other options are emerging in people's values.

The emergence of social networks as a meeting point between people determines fears and judgments, sometimes hurried. Thus come to realize that college students are not a good choice, when in reality for this audience the network is also attractive. They do not go to the networks for their academic inquiries, but without coming within these, if they are for personal and satisfactions to search some of their needs.

Key words: infovirtual, virtual world, social network, network society, virtuality. 


\section{El mundo de las redes}

Los medios de Internet han definido lugares no territoriales de comunicación y encuentro. En este desarrollo la tecnología ha hecho posible el hipertexto, la hipermedia, la interacción en tiempo real, con el agregado especial de la accesibilidad y movilidad de los dispositivos. De aquí que cada vez es más fácil navegar en la red y por supuesto, más atractivo. Pero además, los medios tradicionales como la telefonía o los audiovisuales se han integrado también con tecnologías como voz sobre IP.

Así las cosas, junto con la plaza pública, la institución educativa, el recinto familiar o el ambiente laboral, para la sociedad se presenta de cerca en el ciberespacio otra posibilidad de estar con los demás. Inclusive, las personas pueden estar conectadas al mismo tiempo con estos distintos escenarios. Internet tiene esa bondad, se posibilita cierta manera de ubicuidad, su ímpetu tiene la capacidad de hacer invisibles las fronteras. Con Internet el estar no implica unicidad espacial, esto es, presencia circunscrita únicamente a un contorno, sino que por el contrario, se puede estar virtualmente en otras circunstancias y escenas. La virtualización se concibe como un vector de la creación de realidad (Lévi, 1999).

A lo anterior se suma que el alojamiento de Internet trascendió del computador tradicional a los dispositivos móviles. O sea que el correo electrónico y el vídeo-chat por ejemplo, están en las manos de la gente, donde ellos vayan. Entonces, las personas viajan conectadas, están en red a toda hora. Las personas conviven con Internet en sus proximidades, está ahí en los artefactos electrónicos que fácilmente hacen parte de los utensilios de cada uno.

Pero las personas conviven también con la familia, la institución educativa, los amigos, los medios de comunicación. Estos son los llamados agentes socializadores primarios que influyen fuertemente en las actuaciones posibles (Quintero, 2010). De esta forma puede resultar preocupante que con el ciberespacio se potencie otra resta más a la intimidad de la familia o que los límites escolares sean fácilmente permeados. Ante esto el asunto no es cómo evitar la convivencia en las redes de Internet, pues son tentáculos ya inevitables, sino cómo entender que instantáneamente puede estarse en espacios superpuestos y que así los jóvenes y niños, estando en familia con todos sus valores, pueden estar también con sus amigos en comunión con la certeza de sus identidades. Es decir, las fronteras son franqueables con Internet, es como si se hubieran debilitado las exclusividades de los territorios.

Lo anterior se relaciona con el concepto de la sociedad red, tal como lo ha desarrollado Castells (2002). En esta sociedad el entorno está fundado por multiplicidad de redes de personas y de dispositivos que producen, reciben, procesan, almacenan y transmiten información sin condiciones de distancia, tiempo o cantidad. La gente está en red quiéralo o no. Y las ventajas por pertenecer a más redes sitúan a las personas y las organizaciones en posiciones notables.

La comunicación digital en red es una instancia concluyente para la expansión social, se caracteriza, según Villota, porque rompe fácilmente censuras previas y porque facilita acceso a fuentes especializadas de información y conocimiento. En tal contexto "las tecnologías de la información y la comunicación (TIC) son un "acontecimiento" que se ha hecho cuerpo sin corporeidad, que es real-virtual y que se escribe e inscribe en nuevos textos-hipertextuales. Es decir, la comunicación se ha revertido: es multitemática, integradora, horizontal, cotidiana. De tal forma que la lectura propone delinear esquemas de comprensión en el uso de las TIC, entendidas como instrumento de socialización" (Villota, 2006: p 14).

Una de las realidades del siglo XXI es la expresión de las redes. Las redes de la informática, cada vez más poderosas en cuanto a capacidad de transmisión, determinan industrias especializadas de alta rentabilidad; la convergencia de las comunicaciones integra abanicos de recursos para los usuarios; las comunicaciones satelitales permitan que fácilmente se levanten fuentes de información dispersas en amplias geografías. Desde el punto de vista social, las personas y las empresas tiene oportunidades de competencia y productividad, gracias a las oportunidades de permanecer en distintas conexiones y humanamente, para la in-

Anagramas, Volumen 9, № 17, pp. 107-116 - ISSN 1692-2522 - Julio-diciembre de 2010. 166 p. Medellín, Colombia 
tegridad del ser, las potencialidades se evidencian para asuntos de socio-afectividad, pertenencia, identidad y necesidades.

El desarrollo de Internet ha resultado determinante para esta sociedad red. Especialmente deben considerarse su democratización, su capacidad de integración de distintos medios, su capacidad para almacenar y desplegar conocimientos e información de todo tipo y la oportunidad para que los usuarios adquieran distintos roles, como productores y como receptores de ideas. Además, el poder transcultural, diversificado, potenciado para todo público, abierto, hace que Internet defina la posibilidad del ciberespacio, como una gran red de redes.

\section{Las relaciones sociales en Internet}

Claro está: en el ciberespacio como en el mundo cotidiano, es natural que haya sitios para perseguir fines distintos. O sea que las personas pueden en ejercicio de su libertad ir a lugares físicos o virtuales construidos para el ocio, la diversión o el juego; para el chantaje o la estafa; para la academia, o la ciencia; para aprender o para comunicarse. Según lo anterior, las posibilidades de conductas perniciosas no son exclusividad de Internet, sino alternativas del propio mundo.

Internet da la posibilidad a las personas aisladas por circunstancias propias o externas a ellas, de comunicarse o de pertenecer a grupos sociales. Esta es de las características que más atractiva hacen a esta red y según se observa, cierto publico de su sus comunidades son personas con asilamientos de algún tipo. Pero es de advertirse que tal característica no es una generalización, pues los individuos comúnmente notorios encuentran también en la Red la posibilidad de serlo aun más. De esta forma surgen las comunidades sociales en el ciberespacio.

Aunque no hay referentes exactos, de las observaciones diarias se deducen relaciones entre estos grupos sociales y las extensiones en el espacio físico. Se sabe que de las interacciones, acuerdos o motivaciones de grupos con residencias en el ciberespacio, se determinan acciones en el mundo físico. Y viceversa, los grupos que han surgido allí, empoderados por relaciones entre individuos, por relaciones de convivencia, de compartimento de necesidades, intereses, problemas comunes, encuentran fortalecimiento con las bondades de Internet para sus comunicaciones y compartimientos de información. A propósito de esto, Smith y Kollack (2003: p42) plantean que "las comunidades raramente existen de forma exclusiva en el ciberespacio. Es importante investigar la forma en que los grupos sociales creados en el ciberespacio se expanden en el mundo real y viceversa".

Sobre la constitución de grupos en el ciberespacio, según Zea y Atuesta (2008:p39), "Aunque las comunidades puedan emerger de y existir dentro de grupos unidos a través de redes de computadores, el enlace técnico electrónico de las personas no es suficiente para crearlas. La comunidad incluye más que el simple intercambio de información: es caracterizada, generalmente, por contratos sociales, reciprocidad y economías".

Según esto, son las afinidades, los intereses mutuos, las identidades colectivas, las que justifican la cohesión en red. El enlace de computadores no es más que el medio; por sí misma la disposición pública de Internet no es suficiente para justificar el encantamiento, esto es, existen otros impulsos no digitales que logran convocar a las personas jóvenes a transgredir fronteras para encontrarse con otros. El origen de estas energías solidarias y fraternales está en la estructura de las personas, en su pasado, sus valores, su familia, sus posturas frente a la vida misma.

Las redes sociales del ciberespacio han proliferado gracias al mundo infovirtual, una nueva instancia de la realidad común. Echavarria (2000) lo llamó mundo inforvirtual, Explica que el hombre en el mundo convive en tres entornos posibles: la naturaleza, la ciudad y el entorno telemático o infovirtual. En cuanto a la naturaleza, el ser humano nace para adaptarse a sus implicaciones, siendo necesario desarrollar mecanismos de percepción, movilidad, nutrición e interrelación. El segundo entorno es cultural, social y urbano, se escenifica en las ciudades y los pueblos. El último es una creación de la tecnología, es un nuevo espacio social, con posibilidades para la comunicación, el placer, la formación; dentro de 
este los objetos y los sujetos son representaciones construidas con la electrónica digital. La virtualidad la define Echavarria como la suma de tecnologías informáticas dirigidas para simular percepciones humanas. Puede decirse, desde esta postura, que la virtualidad no es una negación de la realidad, sino una representación de esta con tecnología informática.

El citado autor denomina infovirtual a ese entorno virtual y lo caracteriza por ser informacional (funcional mediante representaciones digitales), representacional (los objetos y las personas no son tangibles, sino que se representan tecnológicamente), comprimido (los objetos y los sujetos, aun siendo complejos, ocupan unidades mínimas), aéreo (esta en el aire, en el espacio informacional o ciberespacio), multisensorial (pude ser audiovisual o textual), distal (los agentes pueden interactuar aun estando distantes).

Internet es la expresión del mundo infovirtual. Es una experiencia ya cotidiana, es una posibilidad de comunicación y convivencia común. Así entonces la asistencia a las redes sociales es apenas algo natural. Estas redes son medios para la instantaneidad, la actuación en tiempo real, el deleite por superar las distancias, el encantamiento producido por tener de cerca en cualquier momento a los suyos. Estas redes emergen como una posibilidad para el establecimiento de relaciones sociales y contratos entre personas que comparten intereses y necesidades. O sea, Internet deja en escena canales para las necesidades de comunicación de los cibernautas, quienes pueden integrarse a comunidades especiales, fundar otras, ensancharlas y relacionarlas con ideales e identidades individuales y comunes.

\section{Las preferencias por las redes sociales de los estudiantes universitarios ${ }^{\prime}$}

Para indagar sobre el impacto de las redes sociales en estudiantes universitarios, se realizó el

\footnotetext{
El proyecto Impacto y manifestaciones de cultura digital en estudiantes universitarios ha generado diversos artículos en distintas revistas de la comunidad académica colombiana. En este caso, se publican las ideas concernientes el tema de las redes sociales.
}

proyecto El ciberespacio para fines académicos y sociales: tendencias halladas en estudiantes universitarios, cuyos objetivos relacionados con el tema de las redes sociales fueron los siguientes:

a) Conocer frecuencias de uso que hacen los estudiantes de recursos disponibles en Internet para fines académicos y de comunicación.

b) Diferenciar fines y motivaciones de los estudiantes sobre la utilización de recursos disponibles en Internet para fines académicos, comunicacionales y socio-afectivos.

c) Evaluar el nivel de utilización que de las redes sociales hacen los estudiantes.

Se realizó la indagación con el propósito de aportar datos para reflexionar acerca de las distancias entre las críticas por la inutilidad de la inmersión juvenil en la Web, y las observaciones que se pronuncian en hábitos motivados por necesidades de información y comunicación. Es decir, cuando no se entiende que las personas jóvenes, al haber nacido en un mundo digital actúan influenciados por sus condiciones, entonces se cae en temores que relacionan las horas de navegación en la red con aconteceres nocivos y a veces peligrosos. Esto puede ser cierto pero es impreciso y parcializado. Inclusive, tales relaciones son injustas al generalizarse para estigmatizar al joven cibernauta.

Claro está: en la red como en el mundo cotidiano, es natural que haya sitios para perseguir fines distintos. O sea que las personas pueden en ejercicio de su libertad ir a lugares físicos o virtuales construidos para el ocio, la diversión o el juego; para el chantaje o la estafa; para la academia, o la ciencia; para aprender o para comunicarse. Según lo anterior, las posibilidades de conductas perniciosas no son exclusividad de Internet, sino alternativas del propio mundo.

Para comprender mejor las aseveraciones sobre la navegación en la red, los datos recogidos entregaron información acerca de los distintos motivos de los jóvenes para ir allí, centrándose especialmente en los propósitos académicos. 
Se tomó una muestra de 940 estudiantes de las siguientes instituciones universitarias de la Ciudad de Medellín: Institución Universitaria Tecnológico de Antioquia, Fundación Universitaria Luis Amigo y Universidad de Antioquia, a inicios del año 2009.

Los estudiantes de la Luis Amigó son de los programas de Ingeniería de Sistemas, Psicología, Derecho, Publicidad, Administración, Contaduría, Comunicación Social, Negocios Internacionales y Educación. En el Tecnológico de Antioquia la muestra se tomó de los programas de Tecnología en Sistemas, Tecnología en Gestión Comercial, Tecnología en Comercio Exterior, Tecnología en Gestión Financiera, Tecnología en Investigación Judicial y Licenciatura en Educación Preescolar.

Por su parte, los estudiantes de la Universidad de Antioquia son de los programas de Ingeniería Industrial y de Ingeniería de Telecomunicaciones.

Se aplicó un método de muestreo no probabilístico por cuotas, con la consideración de tener representación de los siguientes estratos: estudiantes de carreras de ingeniería y tecnología en sistemas y estudiantes de carreras distintas a estas. En este caso fue necesario dividir la población de referencia de acuerdo con la variable tipo de carrera.

Dos de las hipótesis planteadas fueron las siguientes: a) La utilización que de los recursos disponibles en Internet para fines académicos hacen los estudiantes, es menor que la utilización que hacen para fines sociales y de entretenimiento. b) En su mayoría, los estudiantes participan en redes sociales para fines que no son académicos.

Los resultados obtenidos fueron los siguientes:

1) Frecuencia de uso de las redes sociales

\begin{tabular}{|l|c|c|}
\hline \multicolumn{1}{|c|}{ Red } & $\begin{array}{c}\text { Estudiantes } \\
\text { de ingeniería y } \\
\text { tecnología sistemas }\end{array}$ & $\begin{array}{c}\text { Estudiantes diferentes } \\
\text { a ingeniería y } \\
\text { tecnología sistemas }\end{array}$ \\
\hline Facebook & $80 \%$ & $97.8 \%$ \\
\hline MySpace & $24 \%$ & $31.2 \%$ \\
\hline Twitter & $14 \%$ & $16.7 \%$ \\
\hline Fotolog & $2 \%$ & $0.7 \%$ \\
\hline Hi5 & $42 \%$ & $53.6 \%$ \\
\hline
\end{tabular}

\begin{tabular}{|l|c|c|}
\hline \multicolumn{1}{|c|}{ Red } & $\begin{array}{c}\text { Estudiantes } \\
\text { de ingeniería y } \\
\text { tecnología sistemas }\end{array}$ & $\begin{array}{c}\text { Estudiantes diferentes } \\
\text { a ingeniería y } \\
\text { tecnología sistemas }\end{array}$ \\
\hline Sonico & $30 \%$ & $32.6 \%$ \\
\hline $\begin{array}{l}\text { Otras redes } \\
\text { sociales }\end{array}$ & $12 \%$ & $23.2 \%$ \\
\hline
\end{tabular}

Fuente: elaboración propia

Las redes son escenarios para la interacción social, definidas desde un intercambio dinámico entre personas en la web. Se caracterizan por ser grupos de personas que se identifican por las mismas necesidades y problemáticas. Es tan ava sallante su impacto, que para los nativos digitales (personas menores de 25 años) su pertenencia a estas redes puede considerarse uh asunto ya natural. Las cifras así lo revelan, pues el 97\% de los estudiantes han interactuado con Facebook, sin que esta sea la única red, pues en la web hay otras con buena representación.

Llama la atención en los datos anteriores, que los porcentajes de utilización de redes sociales son más bajos en estudiantes de ingeniería y tecnología en sistemas, lo que puede relacionarse con aspectos socio-afectivos relacionados con el tipo de carrera profesional que estudia una persona. Podría deducirse que las personas sumergidas en ámbitos más técnicos $\mathrm{y}$ racionales tienen menos tendencias a socializarse en red, que otras personas de carreras de tipo humano y social.

2) Motivaciones para participar en redes sociales

\begin{tabular}{|l|c|c|}
\hline \multicolumn{1}{|c|}{ Motivos } & $\begin{array}{c}\text { Estudiantes } \\
\text { de ingeniería } \\
\text { y tecnología } \\
\text { sistemas }\end{array}$ & $\begin{array}{c}\text { Estudiantes } \\
\text { diferentes a } \\
\text { ingeniería y } \\
\text { tecnología sistemas }\end{array}$ \\
\hline $\begin{array}{l}\text { Educación y } \\
\text { aprendizaje }\end{array}$ & $40 \%$ & $42.8 \%$ \\
\hline Entretenimiento & $58 \%$ & $81.2 \%$ \\
\hline
\end{tabular}




\begin{tabular}{|l|c|c|}
\hline \multicolumn{1}{|c|}{ Motivos } & $\begin{array}{c}\text { Estudiantes } \\
\text { de ingeniería } \\
\text { y tecnología } \\
\text { sistemas }\end{array}$ & $\begin{array}{c}\text { Estudiantes } \\
\text { diferentes a } \\
\text { ingeniería y } \\
\text { tecnología sistemas }\end{array}$ \\
\hline Curiosidad & $48 \%$ & $65.9 \%$ \\
\hline $\begin{array}{l}\text { Actualidad } \\
\text { noticiosa }\end{array}$ & $26 \%$ & $44.2 \%$ \\
\hline $\begin{array}{l}\text { Ganar } \\
\text { amistades }\end{array}$ & $22 \%$ & $57.2 \%$ \\
\hline
\end{tabular}

Fuente: elaboración propia.

Según estos datos, la curiosidad y el entretenimiento son los motivos que más esgrimen quienes visitan las redes sociales y corroboran las hipótesis sobre su uso. Es decir, este resultado está acorde con lo que la gente normalmente piensa sobre el uso de las redes. Pero hay un elemento que vale destacar: las motivaciones para la educación y el aprendizaje tienen una buena representación, pues los porcentajes son iguales o superiores al $40 \%$.

3) Utilización de recursos de comunicación tipo Web

\begin{tabular}{|c|c|c|}
\hline \multirow{2}{*}{$\begin{array}{c}\text { Recur } \\
\text { so }\end{array}$} & $\begin{array}{c}\text { Estudiantes de } \\
\text { ingeniería y tecnología } \\
\text { sistemas }\end{array}$ & $\begin{array}{c}\text { Estudiantes diferentes } \\
\text { a ingeniería y } \\
\text { tecnología sistemas }\end{array}$ \\
\cline { 2 - 3 } & SI los ha utilizado & SI los ha utilizado \\
\hline Blogs & $44 \%$ & $52.2 \%$ \\
\hline Skipe & $46 \%$ & $52.9 \%$ \\
\hline
\end{tabular}

Fuente: elaboración propia

La comunicación instantánea vía Skipe y los blogs son dos de las aplicaciones en Internet que más impacto han creado dentro de la sociedad, ya que de una manera inmediata y sin mayores dificultades les permiten a las personas ponerse en contacto con el entorno, sin importar las distancias. Estas cifras lo que hacen es demostrar que dentro de la cultura digital de los estudiantes, estas dos aplicaciones tiene también plena vigencia.
4) Número de cuentas de correo electrónico que tienen los estudiantes

\begin{tabular}{|l|c|c|}
\hline Número de cuentas & $\begin{array}{c}\text { Estudiantes de } \\
\text { ingeniería y tec- } \\
\text { nología sistemas }\end{array}$ & $\begin{array}{c}\text { Estudiantes } \\
\text { diferentes a } \\
\text { ingeniería y } \\
\text { tecnología sistemas }\end{array}$ \\
\hline Una & $14 \%$ & $46.3 \%$ \\
\hline Dos & $38 \%$ & $44.1 \%$ \\
\hline Tres o más & $36 \%$ & $8.4 \%$ \\
\hline $\begin{array}{l}\text { No tiene cuenta } \\
\text { de correo }\end{array}$ & $0 \%$ & $0 \%$ \\
\hline
\end{tabular}

Fuente: elaboración propia.

De los anteriores, un dato es sorprendente y es el relacionado con las personas que no tienen cuenta de correo electrónico. De los encuestados todos los estudiantes tienen correo electrónico y en el caso de los estudiantes de ingeniería y tecnología en sistemas el porcentaje de quienes tienen dos, tres o más cuentas de correo es significativo.

5) Frecuencia de uso del correo electrónico

\begin{tabular}{|l|c|c|}
\hline Número de cuentas & $\begin{array}{c}\text { Estudiantes } \\
\text { de ingeniería } \\
\text { y tecnología } \\
\text { sistemas }\end{array}$ & $\begin{array}{c}\text { Estudiantes } \\
\text { diferentes a } \\
\text { ingeniería y } \\
\text { tecnología sistemas }\end{array}$ \\
\hline Una vez en el día & $8 \%$ & $26.8 \%$ \\
\hline Varias veces en el día & $58 \%$ & $47.1 \%$ \\
\hline Ocasionalmente & $8 \%$ & $35.5 \%$ \\
\hline Nunca lo utiliza & $0 \%$ & $0 \%$ \\
\hline
\end{tabular}

Fuente: elaboración propia

Según estos datos, todos los estudiantes utilizan el correo electrónico y los porcentajes más altos están representados por estudiantes que utilizan este medio varias veces en el día.

6) Utilización de la red social Facebook

\begin{tabular}{|c|c|c|}
\hline \multirow{2}{*}{ Propósito } & $\begin{array}{c}\text { Estudiantes de ingeniería y } \\
\text { tecnología sistemas }\end{array}$ & $\begin{array}{c}\text { Estudiantes } \\
\text { diferentes a ingeniería } \\
\text { y tecnología sistemas }\end{array}$ \\
\cline { 2 - 3 } & SI ha conseguido amigos & $\begin{array}{c}\text { SI ha conseguido } \\
\text { amigos }\end{array}$ \\
\hline Amistad & $40 \%$ & $79 \%$ \\
\hline
\end{tabular}




\begin{tabular}{|l|c|c|}
\hline \multirow{2}{*}{ Propósito } & $\begin{array}{c}\text { Estudiantes } \\
\text { de ingeniería y } \\
\text { tecnología sistemas }\end{array}$ & $\begin{array}{c}\text { Estudiantes } \\
\text { diferentes a } \\
\text { ingeniería y } \\
\text { tecnología sistemas }\end{array}$ \\
\cline { 2 - 3 } & $\begin{array}{c}\text { Si tiene este } \\
\text { propósito }\end{array}$ & $\begin{array}{c}\text { Si tiene este } \\
\text { propósito }\end{array}$ \\
\hline Afecto & $6 \%$ & $5.1 \%$ \\
\hline Diversión & $76 \%$ & $79.7 \%$ \\
\hline Desahogo & $16 \%$ & $0 \%$ \\
\hline
\end{tabular}

Fuente: elaboración propia.

Facebook y Google son las dos aplicaciones en Internet que más han transformado las formas de participación de las personas y que con mayor vigor ha contribuido su impacto social. Es así como para los jóvenes y niños prácticamente el mundo es inimaginable sin las potencialidades de estos dos recursos públicos, pues ellos hacen fácil el establecimientos de comunicaciones, la pertenencia, la identidad grupal, la libertad de actuación y la expresividad.

Con respecto a Facebook, comúnmente dentro de la gente se encuentran aseveraciones acerca de los propósitos de esta red social para que sus actores encuentren en su maraña caminos hacia el afecto, la diversión y el desahogo. Pero los resultados de la encuesta no respaldan en su totalidad estas hipótesis, pues los propósitos de afecto y desahogo no están representados con porcentajes significativos. En cambio los propósitos de amistad y diversión sí muestran unos resultados visibles.

Medios utilizados para la comunicación en Internet

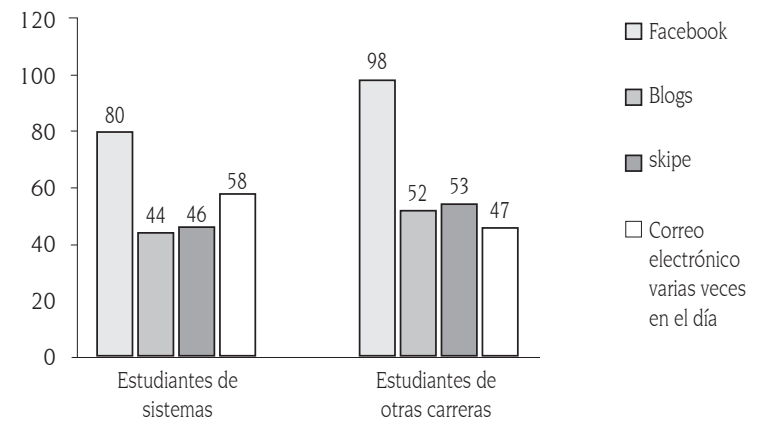

Grafico $N^{\circ} 1$.

Fuente: elaboración propia.
Facebook sobresale por la aceptación que tiene entre los jóvenes. Estos datos no hacen más que demostrar fehacientemente el impacto de esta red social, la cual no solamente es preferida como espacio virtual para encuentro de amigos, sino como medio para hacer mercadeo, generar negocios, divulgar noticias, emprender campañas o hacer denuncias. Como red pública social, libre, democrática, de fácil uso y penetración, está expuesta al uso delictivo o dañino de quienes así lo determinen. Así las cosas, Facebook como escenario está expuesto en el ciberespacio y son la moral y la ética de los individuos quienes determinan los propósitos de sus usos.

La red social Facebook es el medio para la comunicación más utilizado en Internet, según esto datos. Llama la atención que la preferencia entre los estudiantes de otras carreras alcance el 98\%, lo cual demuestra prácticamente una cobertura total. Esto coincide con la percepción general sobre los hábitos de los jóvenes y adolescentes. Ellos están extendiendo su vida cotidiana a esa red, la convivencia allí es natural, en cuanto a que las necesidades de comunicación y reconocimiento encuentran respuestas inmediatas. .

Con los resultados obtenidos se logró la siguiente contrastación de hipótesis:

Primera hipótesis: La utilización que de los recursos disponibles en Internet para fines académicos hacen los estudiantes es menor que la utilización que hacen para fines sociales y de entretenimiento.

En cuanto a la utilización de vídeos, las diferencias porcentuales entre las motivaciones para su utilización son estrechas. Los estudiantes utilizan los vídeos con fines de aprendizaje, entretenimiento y curiosidad en similar proporción, y en menor para actualización noticiosa.

Este resultado deja dudas sobre los argumentos que ponen el uso de Internet como un cataclismo. Se trata, desde luego, de preocupaciones que emergen a partir del masivo uso de la red, que incluso alcanzan a conceptualizaciones no muy claras como "adicciones a Internet" (Balaguer, 2003), en referencia a la afición al ciberespacio.

Por supuesto, el uso de Internet entre los jóvenes puede considerarse un esnobismo. Sin 
embargo, puede ser también un asunto de necesidad de comunicación. Pero sea lo uno o lo otro, en realidad la nocividad no es absoluta o ni tan directamente evidente. Por lo siguiente: los vídeos son de los objetos más perseguidos por los cibernautas jóvenes, y según los resultados de esta investigación, son apetecidos tanto para fines de aprendizaje como para entretenimiento y curiosidad. Pero, además, no todo afán por estos dos motivos es siempre pernicioso, pues hay diversiones sanas y juegos lícitos.

Segunda hipótesis: En su mayoría, los estudiantes participan en redes sociales para fines que no son académicos.

Al comparar este enunciado con los resultados obtenidos, se deduce la contrastación positiva (participación con fines no académicos), dado que el porcentaje de estudiantes que visitan la red Facebook es superior al 80\%, pero para fines de educación y aprendizaje la cifra es inferior al 43\%.

Aunque no se declaran en la formulación de la hipótesis, en el instrumento aplicado los fines no académicos de utilización de las redes sociales están relacionados con entretenimiento, curiosidad, actualidad noticiosa y círculo de amistades. En los estudiantes de carreras distintas a ingeniería y tecnología en sistemas, estas cuatro motivaciones superan a las relacionadas con la educación y el aprendizaje. A su vez, los estudiantes de estas dos carreras expresaron la motivación académica por debajo de la curiosidad y el entretenimiento. Pero además, el 79\%de los estudiantes de carreras distintas a ingeniería y tecnología en sistemas dicen que han conseguido amigos en Facebook. Llama también la atención que más del $75 \%$ de las dos poblaciones analizadas dicen que acuden a la red por diversión.

Ahora bien, la evaluación de estas tendencias dentro del marco de las necesidades humanas.de Maslow (1991) explica cómo dentro de la red los jóvenes pueden encontrar respuesta a necesidades de estima, amor, pertenencia, auto-realización y trascendencia. Estas son realizaciones de las potencialidades de las personas para alcanzar su identidad. Es un problema de identidad humana, del yo, o sea de la configuración humana misma. Es más, desde el punto de vista de Max-Neef
(1986), existen distintos tipos de satisfactores como autonomía, autoestima, receptividad, amistades, cooperación, curiosidad, humor o expresión, que pueden satisfacer las necesidades existenciales (ser, tener, hacer, estar) o axiológicas (subsistencia, protección, afecto, entendimiento, participación, ocio, creación, identidad y libertad).

Según lo anterior, es limitada la visión que relaciona la importancia de Facebook solo con aspiraciones académicas, cognitivas o de aprendizaje, y puede decirse, apresuradas las críticas que pregonan sobre la forma como los jóvenes abusan de esta red. Pueden ser abusos, pero desde una mirada externa porque en el interior del círculo de esos jóvenes en red, puede más bien hablarse sobre usos necesarios. Estas posiciones externas son de quienes no son nativos digitales o de quienes, siéndolo, no usan la red social porque no sienten la necesidad de navegar allí. Situados en los conceptos de Maslow y Max-Nef pueden existir motivaciones no académicas que lleven a los jóvenes a Facebook. Es decir, existen otras necesidades, por ejemplo, ser entendidos, ser escuchados, ser participativos. En estos términos, en las comunidades sociales de Internet, los jóvenes encuentran satisfactores que los conducen a la producción de ideas, al sostenimiento de diálogos de todo tipo, al encuentro de mensajes que coinciden con sus intereses.

\section{Conclusiones}

Las personas del siglo XXI, especialmente los jóvenes, tienen en el ciberespacio una oportunidad más para sus necesidades de comunicación e identidad. Sin embargo, ello no significa ruptura con sus otros espacios.

Lo peculiar de estas opciones es la realización de las redes. Internet convoca a estar en red, su funcionalidad y su misma concepción determinan un mundo infovirtual y obligan a la permanencia en ellas. Aparte de esto, el entorno globalizado se plantea como una sociedad red, lo que significa que los intereses de las empresas y las personas se proyectan y se validan socialmente pero en términos de conectividad con otros nodos y de pertenencia distribuida. 
Otro aspecto a considerarse es la ubicuidad que se permite desde el ciberespacio. Las personas pueden fácilmente estar situadas y, a su vez, estar distantes, esto es, estar aquí y allá. Departir de unos asuntos en un lugar y fugarse en tiempo real a atender otros.

Así las cosas, es fácil comprender la identificación de los jóvenes con las bondades de Internet y más cuando, debido a convergencias de las telecomunicaciones, la red llega a los dispositivos móviles y concentra, en su espacio, posibilidades como la radio, las revistas, la música o los vídeos.

La investigación que se presento así los demostró. Es casi generalizado el uso que de las redes sociales de Internet hacen los jóvenes universitarios. Ellos aun con sus intereses académicos van a estas comunidades no exactamente a eventos de ese tipo. Ellos van a estas redes a satisfacer necesidades de pertenencia, diversión o simplemente, por curiosidad. Lo cierto es que este entorno no es ajeno a ellos.

\section{Bibliografía}

Balaguer, R. (2003). Internet: un nuevo espacio psicosocial. Montevideo: Ediciones Trilce.

Castells, M. (2002). La era de la información. Vol. I: La Sociedad Red. México: Siglo XXI Editores.

Echeverría, J. (2000). Un mundo virtual. Barcelona: Plaza $\varepsilon$ Janes Editores S. A.

Lévy, P. (1999). ¿Qué es la virtualidad? Barcelona: Paidós.

Maslow, A. (1991). Motivación y personalidad. Madrid: Díaz De Santos.

Max-Neef, M. (1986). Desarrollo a escala humana. Conceptos, aplicaciones y algunas reflexiones. Montevideo: Nordan-Comunidad.

Quintero, C. (2010). Rasgos significativos en la adolescencia. Espacio Educativo, 11. Bogotá: CEDAL.

Villota, O. (2006). Redes del conocimiento y la creatividad. Cultura mediática. Internet y Televisión. Crítica y creatividad. Bogotá: CEDAL.

Zea, C. y Atuesta, R. (2008) Hacia una comunidad interactiva educativa. Medellín: Fondo Editorial Universidad Eafit. 